OPEN ACCESS

Edited by:

Marco Rinaldo Oggioni, University of Leicester,

United Kingdom

Reviewed by:

Jie Wen,

Institute of Animal Sciences (CAAS),

China

Annalisa Ciabattini, University of Siena, Italy

Janet Yakubu Nale,

University of Leicester,

United Kingdom

${ }^{*}$ Correspondence:

Hai Lin

Hailin@sdau.edu.cn

Shuhong Sun

jqybfkyjs@163.com

Specialty section: This article was submitted to Microbial Immunology, a section of the journal

Frontiers in Microbiology

Received: 14 October 2019

Accepted: 10 February 2020

Published: 26 February 2020

Citation:

Zhao X, Yang J, Ju Z, Wu J, Wang L, Lin H and Sun S (2020) Clostridium butyricum Ameliorates

Salmonella Enteritis Induced

Inflammation by Enhancing and Improving Immunity of the Intestinal Epithelial Barrier at the Intestinal

Mucosal Level.

Front. Microbiol. 11:299.

doi: 10.3389/fmicb.2020.00299

\section{Clostridium butyricum Ameliorates Salmonella Enteritis Induced Inflammation by Enhancing and Improving Immunity of the Intestinal Epithelial Barrier at the Intestinal Mucosal Level}

\author{
Xiaonan Zhao ${ }^{1,2}$, Jie Yang ${ }^{1}$, Zijing Ju' ${ }^{1}$, Jianmin Wu' ${ }^{1}$, Lili Wang ${ }^{1}$, Hai Lin ${ }^{1 *}$ and \\ Shuhong Sun ${ }^{1 *}$
}

${ }^{1}$ College of Animal Science and Technology, Shandong Agricultural University, Tai'an, China, ${ }^{2}$ Institute of Animal Science and Veterinary Medicine, Shandong Academy of Agricultural Sciences, Jinan, China

This study was aimed to investigate the effects of Clostridium butyricum (C. butyricum) immunity and intestinal epithelial barrier function at the intestinal mucosal level, by using Salmonella enteritidis (S. enteritidis) to infect specific-pathogen-free (SPF) chickens and intestinal epithelial cells (IEC). We found that $C$. butyricum could decrease cytokine levels (IFN- $\gamma, \mathrm{IL}-1 \beta, \mathrm{IL}-8$, and TNF- $\alpha$ ) via the TLR4-, MyD88-, and NF-kB-dependent pathways in intestinal tissues and intestinal epithelial cells. Additionally, C. butyricum could attenuate bacteria-induced intestinal damage and increase the expression level of muc-2 and ZO-1 in the intestine and intestinal epithelial cells. Furthermore, C. butyricum altered the intestinal microbial composition, increased the diversity of the bacterial communities in the cecum of Salmonella-infected chickens. In conclusion, C. butyricum effectively attenuated inflammation and epithelial barrier damage, altered the intestinal microbial composition, increased the diversity of the bacterial communities in the intestine of Salmonella-infected chickens. The result suggests that $C$. butyricum might be an effective and safe therapy for the treatment of Salmonella infection.

Keywords: C. butyricum, Salmonella enteritidis, immunity, intestine, intestinal microflora

\section{INTRODUCTION}

Salmonella is a common bacterial entero-pathogen and one of the leading causes of serious illness in humans and animals, such as enteritis and diarrhea (Mathur et al., 2012). Over 20 million individuals suffer from typhoid fever, and more than 220,000 deaths each year have been reported around the world (Majowicz et al., 2010; Feasey et al., 2012).

Chickens have been recognized as an important reservoir for Salmonella (Chen and Jiang, 2014). The most frequently isolated serovar from chickens is $S$. enteritidis (Zhao et al., 2017). After oral ingestion in chickens, Salmonella initially breaches the epithelial lining, which is the first line of defense against the invasion of microbes and their associated lipopolysaccharide (LPS) and toxins. Impaired epithelial barrier function may predispose to various intestinal disorders, such as inflammation (Juan et al., 2018; Xiao et al., 2018). In addition, Mucins are the 
primary conpinents of intestinal mucus layer that are part of the innate immune system and act as a barrier against luminal pathologies (Forstner et al., 1995; Huang et al., 2015).

In recent years, antibiotics have been effectively used to treat Salmonella infection. Unfortunately, the widespread use of antibiotics has increased bacterial resistance and led to intestinal flora imbalance, which considerably diminish the efficacy of chemical antibiotics (Parry and Threlfall, 2008). Alternatively, the use of probiotic bacteria can modulate systemic and mucosal immune function, improve intestinal barrier function, alter gut micro-ecology, induce secretion of cytokines and Ig in serum, and perturb the MyD88 signaling pathway (Kusumawati et al., 2006; Shanahan, 2010; Madsen, 2012; Kemgang et al., 2014; Lim et al., 2017).

Clostridium butyricum is a gram-positive, obligate anaerobe and endospore-forming probiotic, which has been widely used for repairing intestinal epithelium, thereby improving gastrointestinal function (Cao et al., 2012). A preliminary study demonstrated that $C$. butyricum could reduce the colonization of pathogenic bacteria, weakening the inflammatory response (Zhang et al., 2016). However, the mechanism of protection remains to be elucidated.

In this study, we aimed to explore the mechanism by which C. butyricum could suppress the pathogenic strain $S$. enterica using the specific-pathogen-free (SPF) chicken model with an emphasis on the response at the intestinal mucosal level.

\section{MATERIALS AND METHODS}

\section{Ethics Statement}

All procedures were approved by the Animal Care and Use Committee of Shandong Agricultural University (SDAUA-2016016), and all husbandry practices and euthanasia were performed with full consideration of animal welfare.

\section{Bacterial Strains}

Clostridium butyricum (AQQF01000149) was obtained from Dalian Sanyi Animal Medicine Company (China). The strain was cultured anaerobically with Reinforced Clostridial Medium (RCM) broth at $37^{\circ} \mathrm{C}$ for $48 \mathrm{~h}$. According to the plate count method as described by Wei et al. (2013), the concentration of the bacteria was adjusted to $10^{6}$ colony forming units $(\mathrm{CFU}) / \mathrm{mL}$.

A virulent atrichia strain of $S$. enteritidis was obtained from the Avian Disease Centre of Shandong Agricultural University, and it was selected for the challenge study due to the invasive characteristic previously described (Zhao et al., 2017). The S. enteritidis strain was cultured with nutrient broth at $37^{\circ} \mathrm{C}$ for $12 \mathrm{~h}$. To eliminate the possible LPS contamination, $S$. enteritidis cells were collected by centrifugation at 7,000 $\times g$ for $10 \mathrm{~min}$ and washed twice with PBS ( $\mathrm{pH} 7.2$ ), followed by dilution with PBS to a final cell count of $10^{6}$ colony forming units (CFU)/mL according to the LD50.

\section{Animals}

Specific-pathogen-free chickens were obtained from Jinan SPAFAS Poultry Company (Jinan, China). SPF chickens refer to animals that do not have specific microorganisms or parasites, but may carry non-specific microorganisms and parasites, also known as third-class animals (The European Pharmacopoeia 7. 0, 2010). Chickens were reared in the animal room of Shandong Agricultural University. Chickens were reared in metal cages, and the temperature was maintained at $30^{\circ} \mathrm{C}$ for the first 3 days and gradually reduced to $28^{\circ} \mathrm{C}$ during the last days of the experiment. Chickens were fed with a commercial diet and had free access to feed and water during the whole experimental period. The nutrient levels of the basal diet met the nutritional requirement of the chickens (NRC, 1994) (Table 1). At 1 and 7 days of age, birds were tested for the absence of Salmonella by taking cloacal swabs. Thereafter, a total of 60 health chickens were randomly assigned to three groups ( $n=20$ /group): (Mathur et al., 2012) orally administered $0.2 \mathrm{~mL}$ sterile saline solution per chicken once every day from day 1 through day 14 [negative control group (NC)]; (Feasey et al., 2012) orally administered $0.2 \mathrm{~mL}$ sterile saline solution per chicken once every day from day 1 through day 14 and challenged with $0.2 \mathrm{~mL} S$. enteritidis $\left(10^{6} \mathrm{CFU} / \mathrm{mL}\right)$ on day 8 [S. enteritidis infected group, positive control (PC)]; and (Majowicz et al., 2010) orally administered $0.2 \mathrm{~mL}$ C. butyricum $\left(10^{6} \mathrm{CFU} / \mathrm{mL}\right)$ once every day from day 1 through day 14 and challenged with $0.2 \mathrm{~mL} \mathrm{~S}$. enteritidis $\left(10^{6} \mathrm{CFU} / \mathrm{mL}\right)$ on day 8 [C. butyricum $+S$. enteritidis treatment (EXP)]. At the age of 14 days ( $6 \mathrm{dpi})$, all birds were euthanized via cervical dislocation. The tissues of duodenum, jejunum, ileum, and cecum were collected and stored in liquid nitrogen for mRNA and histological analysis. The cecal contents were collected and stored at $-80^{\circ} \mathrm{C}$ for microbial composition analysis.

\section{Histological Study of the Cecum}

One inch of the cecum of chickens was removed, fixed in $4 \%$ paraformaldehyde and prepared for histological studies as described by Sainte-Marie (1962). Paraffin sections of $5 \mu \mathrm{m}$ were deparaffinized in xylene and stained with hematoxylin and eosin

TABLE 1 | The composition and nutrients of basal diet.

\begin{tabular}{lclc}
\hline Ingredient & Content (\%) & Chemical composition & Content \\
\hline Corn & 55.23 & CP,\% & 20.90 \\
Soybean meal & 30.67 & ME, Mcal/kg & 3.00 \\
Wheat shorts & 4.00 & Calcium,\% & 1.00 \\
Fish meal & 3.00 & Total P,\% & 0.65 \\
Soybean oil & 2.90 & Available P,\% & 0.45 \\
DL-Methionine & 0.27 & Methionine + cysteine,\% & 0.90 \\
NaCl & 0.27 & Lysine,\% & 1.05 \\
Limestone & 1.33 & & \\
Calcium phosphate & 1.33 & & \\
Vitamin-mineral premix & 1.00 & & \\
\hline
\end{tabular}

${ }^{a}$ Crude protein content is $62.5 \%$ and metabolizable energy is $2.79 \mathrm{Mcal} / \mathrm{kg}$. ${ }^{b}$ Metabolizable energy is $8.8 \mathrm{Mcal} / \mathrm{kg}$. ' Supplied per kilogram of diet: vitamin A (retinyl acetate), 1,500 IU; cholecalciferol, 200 IU; vitamin E (DL- $\alpha$-tocopheryl acetate), $10 \mathrm{U}$; riboflavin, $3.5 \mathrm{mg}$; pantothenic acid, $10 \mathrm{mg}$; niacin, $30 \mathrm{mg}$; cobalamin, $10 \mathrm{ug}$; choline chloride, $1,000 \mathrm{mg}$; biotin, $0.15 \mathrm{mg}$; folic acid, $0.5 \mathrm{mg}$; thiamine $1.5 \mathrm{mg}$; pyridoxine $3.0 \mathrm{mg} ; \mathrm{Fe}, 80 \mathrm{mg}$; Zn, $40 \mathrm{mg}$; Mn, $60 \mathrm{mg}$; $0.18 \mathrm{mg}$; $\mathrm{Cu}, 8 \mathrm{mg}$; Se, $0.15 \mathrm{mg}$. 
(H\&E) for microscopic examination, and the overall quality of villi was observed.

\section{Microbial Composition Analysis}

$100 \mathrm{mg}$ cecum contents samples were collected and microbial genomic DNA was extracted from cecum contents using TIANamp Stool DNA Kit (Tiangen, Beijing, China) according to the manufacturer's instructions. The V4 hypervariable region of the $16 \mathrm{~S}$ rRNA gene was amplified by PCR using $515 \mathrm{~F}$ and 806R primers. Eighteen samples ( $n=6$ /group) were sequenced on an Illumina MiSeq platform provided by Personalbio (Shanghai, China). Paired-end reads from the original DNA fragments were merged using FLASH. Clustering was performed using the UPARSE pipeline, and sequences were assigned to operational taxonomic units at $97 \%$ similarity (Schloss et al., 2009). The diversity and composition of the bacterial community was determined by $\alpha$ diversities according to Personalbio's recommendations. The Chaol and ACE indexes simply refer to the number of species in the community, regardless of the abundance of each species in the community, the Shannon's diversity index considers both richness and evenness, the higher Chao1, ACE and Shannon index are, the higher the species diversity are.

\section{Real-Time PCR}

Total RNA was extracted from duodenal, jejunal, ileal, and cecal tissues using Trizol reagent (Invitrogen, United States) according to the manufacturer's instructions. Briefly, 50-100 mg tissue samples were ground to powder with liquid nitrogen and transferred to a tube with $1 \mathrm{ml}$ of Trizol; after centrifuged at $4^{\circ} \mathrm{C}, 0.2 \mathrm{ml}$ chloroform was added to the supernatant; after centrifuged at $4^{\circ} \mathrm{C}$, the supernatant containing the intact RNA was transferred to a new tube, RNA was then precipitated with equal volume of isopropyl alcohol, and washed with $80 \%$ ethanol. The RNA was solubilized in RNase free water. RNA quantity and quality were evaluated using a NanoDrop ${ }^{\mathrm{TM}} 2000$ spectrophotometer (Thermo Fisher Scientific, Waltham, MA, United States), followed by cDNA synthesis via the Transcriptor First-Strand cDNA Synthesis Kit (Roche, China) using $2 \mu \mathrm{g}$ RNA template. Real-time PCR was performed using SYBR Green I Master mix (Roche). Two microliters of cDNA, $5 \mu 1$ SYBR Green buffer $2 \times$ (Roche) and 2.5 pmol of each primer were combined for a total reaction volume of $10 \mu \mathrm{l}$. The thermocycler protocol consisted of a $5 \mathrm{~min}$ pre-incubation at $95^{\circ} \mathrm{C}$ for $20 \mathrm{~s}, 60^{\circ} \mathrm{C}$ for $30 \mathrm{~s}$ and $72^{\circ} \mathrm{C}$ for $20 \mathrm{~s}$, and melt curves were added. The $\beta$-actin reference gene was chosen for the relative expression of targeted genes. mRNA relative expression was calculated using the $2^{-\Delta \Delta}$ ${ }^{\mathrm{Ct}}$ method. The primers used in this study are listed in Table 2.

\section{Primary Chicken Intestinal Epithelial Cell Culture}

Specific-pathogen-free eggs were purchased from Jinan SPAFAS Poultry Company (China). Chicken intestinal epithelial cells (IECs) were prepared from 19-day-old SPF chicken embryos as described previously (Pierzchalska et al., 2012) with some modifications. Briefly, the duodenum was excised, cut into small
TABLE 2 | Primer sequences of targeted and reference genes.

\begin{tabular}{|c|c|c|}
\hline Gene & Sequence $\left(5^{\prime}-3^{\prime}\right)$ & References \\
\hline TLR4 & $\begin{array}{l}\text { Forward: AGTCTGAAATTGCTGAGCTCAAAT } \\
\text { Reverse: GCGACGTTAAGCCATGGAAG }\end{array}$ & Zhao et al., 2017 \\
\hline MyD88 & $\begin{array}{l}\text { Forward: TGATGCCTTCATCTGCTACTG } \\
\text { Reverse: TCCCTCCGACACCTTCTTCTA }\end{array}$ & Zhao et al., 2017 \\
\hline $\mathrm{NF}-\kappa \mathrm{B}$ & Forward: CAGCCCATCTATGACAACCG & Zhao et al., 2017 \\
\hline $\mathrm{IFN}-\gamma$ & $\begin{array}{l}\text { Reverse: TCCCTGCGTCTCCTCTGTGA } \\
\text { Forward: CTGACGGTGGACCTATTATTGTAG } \\
\text { Reverse: GTITGATGTGCGGCTITGA }\end{array}$ & Zhao et al., 2017 \\
\hline $\mathrm{IL}-1 \beta$ & $\begin{array}{l}\text { Forward: GTGAGGCTCAACATTGCGCTGTA } \\
\text { Reverse: TGTCCAGGCGGTAGAAGATGAAG }\end{array}$ & Zhao et al., 2017 \\
\hline IL-8 & $\begin{array}{l}\text { Forward: ATGAACGGCAAGCTTGGAGCTG } \\
\text { Reverse: TCCAAGCACACCTCTCTTCCATCC }\end{array}$ & Zhao et al., 2017 \\
\hline TNF- $\alpha$ & $\begin{array}{l}\text { Forward: TGCTGTTCTATGACCGCC Reverse: } \\
\text { CाTCAGAGCATCAACGCA }\end{array}$ & Zhao et al., 2017 \\
\hline Muc-2 & $\begin{array}{l}\text { Forward: AGGCCAGTTCTATGGAGCACAGTT } \\
\text { Reverse: TTGAGTGCCCAGAGGGACATTTCA }\end{array}$ & Huang et al., 2015 \\
\hline $\mathrm{ZO}-1$ & $\begin{array}{l}\text { Forward:GCGCCTCCCTATGAGGAGCA } \\
\text { Reverse:CAAATCGGGGTTGTGCCGGA }\end{array}$ & Zuo et al., 2014 \\
\hline Occludin & $\begin{array}{l}\text { Forward:TCGTGCTGTGCATCGCCATC } \\
\text { Reverse:CGCTGGTTCACCCCTCCGTA }\end{array}$ & Zuo et al., 2014 \\
\hline$\beta$-Actin & $\begin{array}{l}\text { Forward: GAGAAATTGTGCGTGACATCA } \\
\text { Reverse: CCTGAACCTCTCATTGCCA }\end{array}$ & Zhao et al., 2017 \\
\hline
\end{tabular}

pieces with a sterile scalpel blade, and dissected perpendicularly to expose the lumen. Small duodenal pieces were transferred to a tube filled with DMEM/Ham's/F12 (Gibco, Grand Island, NY, United States) medium with $1 \%$ fetal bovine serum (Gibco), $50 \mu \mathrm{g} / \mathrm{ml}$ gentamycin (Invitrogen, Carlsbad, CA, United States), $100 \mu \mathrm{l} / \mathrm{ml}$ penicillin/streptomycin $(10,000 \mathrm{U} / \mathrm{ml} / 10,000 \mu \mathrm{g} / \mathrm{ml})$ (Invitrogen, Carlsbad, CA, United States), $1 \mathrm{U} / \mathrm{ml}$ dispase II (Roche, Basel, Switzerland) and $75 \mathrm{U} / \mathrm{ml}$ collagenase (Gibco). Digestion was performed at $37^{\circ} \mathrm{C}$ under steady agitation for $2 \mathrm{~h}$. The material was filtered, and larger pieces were discarded, while medium containing single cells and small pieces was centrifuged at $100 \times g$ for $3 \mathrm{~min}$. To separate mucus and IECs, a centrifugation step of $10 \mathrm{~min}$ was performed at $400 \times g$. Mucus covering the cell pellet was discarded. The remaining cell pellet was subsequently washed several times until the suspension was clear, and finally, $1 \times 10^{7}$ cells were cultured in six-well plates and incubated at $37^{\circ} \mathrm{C}$ with $5 \% \mathrm{CO}_{2}$. After incubation for $48 \mathrm{~h}$, IECs were treated under three different conditions as follows: (NC) DMEM alone; (PC) S. enteritidis $\left(10^{6} \mathrm{CFU}\right)$ infection only; and (EXP) pre-incubation with C. butyricum $\left(10^{6} \mathrm{CFU}\right)$ for $2 \mathrm{~h}$ prior to exposure to $S$. enteritidis. At 2 and $6 \mathrm{~h}$ after $S$. enteritidis challenge, a portion of the cells were then collected and treated with lysis buffer to extract total RNA for real-time PCR.

\section{Statistical Analysis}

Statistical evaluations were performed using a one-way ANOVA followed by a Duncan multiple range test or a Fisher least significant difference test using SPSS 16.0 (SPSS, Chicago, IL, United States). Data were visualized using GraphPad Prism 5 software (GraphPad Software, Inc., San Diego, CA, United States). $P<0.05$ was considered significant. 


\section{RESULTS}

\section{C. butyricum Improved Morphology and Integrity in the Cecum}

Microscopic examination revealed that chicken infected with $S$. enteritidis in the PC group showed surface damage and disruption to villi. Cecal tissue of chickens pre-treated with C. butyricum in the EXP group showed less severe surface damage to villi than did cecal tissue of chickens in the PC group. These observations demonstrate that pretreatment of C. butyricum resulted in a reduction of bacteria-induced intestinal damage (Figure 1).

\section{Determination of Cytokine Levels in Intestines}

Cytokine levels were measured to test the hypothesis that early pretreatment of chicken with C. butyricum may alter cytokine production in intestinal tissue following $S$. enteritidis challenge.

The gene expression levels of cytokines IFN- $\gamma$, IL- $1 \beta$, IL8 , and TNF- $\alpha$ in intestinal tissue (i.e. duodenum, jejunum, ileum, and cecum) were also evaluated. The results showed that at 6 days post-infection, no significant differences were found in IFN- $\gamma$ and TNF- $\alpha$ among NC, PC, and EXP groups in intestinal tissue (i.e. duodenum, jejunum, ileum, and cecum) $(P>0.05)$ (Figures 2A,D). The gene expression level of IL$1 \beta$ in the duodenum was significantly elevated in the PC group compared to the NC and EXP group $(P<0.05)$, but there was no significant difference between the NC and EXP groups $(P>0.05)$; in the jejunum, the gene expression level of IL$1 \beta$ was significantly elevated in the PC group compared to the EXP group $(P<0.05)$, but there was no significant difference between the NC and EXP groups and the same change between NC and PC groups $(P>0.05)$; in the ileum, no significant difference of IL- $1 \beta$ was found among NC, PC, and EXP groups $(P>0.05)$; in the cecum, the gene expression level of IL-1 $\beta$ was significantly elevated in the PC group compared to the NC group $(P<0.05)$, but no significant difference was found between the NC and EXP groups and the same change between $\mathrm{PC}$ and EXP groups $(P>0.05)$ (Figure 2B). The gene expression level of IL- 8 in the jejunum was significantly elevated in the PC group compared to the NC and EXP groups $(P<0.05)$, but no significant difference was found between the $\mathrm{NC}$ and EXP groups $(P>0.05)$; of note, no significant difference of
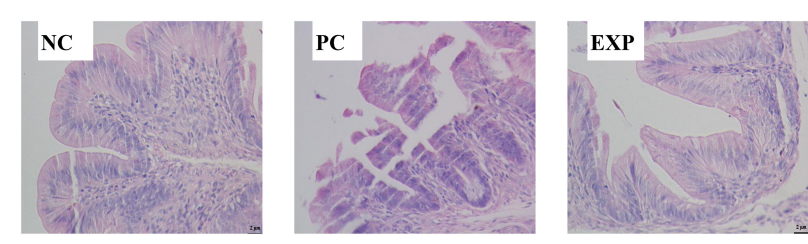

FIGURE 1 | Representative histopathology of cecal tisssues at 6 days post-infection. Three independent experiments showing similar results with 6-8 chicken per treatment. NC, the negative control group; PC, the positive control group; EXP, C. butyricum + S. enteritidis treatment.
IL-8 in duodenum, ileum, and cecum was found among NC, PC and EXP groups $(P>0.05)$ (Figure 2C). Furthermore, we investigated the effects of $C$. butyricum on cytokine expression in IECs in vitro. The results showed that after $2 \mathrm{~h}$ of infection, the expression level of IFN- $\gamma$ was significantly elevated in the PC group compared to the NC and EXP groups $(P<0.05)$, but there was no significant difference between the NC and EXP groups $(P>0.05)$ (Figure 3A). The expression level of IL-8 was significantly elevated in the PC and EXP groups compared to the NC group $(P<0.05)$, but there was no significant difference between the PC and EXP groups $(P>0.05)$. Regarding the expression levels of IL- $1 \beta$ and TNF- $\alpha$, no significant difference was found among the NC, PC, and EXP groups $(P>0.05)$ (Figures 3B-D). After $6 \mathrm{~h}$ of infection, the expression levels of IFN- $\gamma$ and TNF- $\alpha$ were significantly elevated in the PC group compared to the NC and EXP groups $(P<0.05)$, but there was no significant difference between the NC and EXP groups $(P>0.05)$ (Figures 3A,D). The gene expression levels of IL-1 $\beta$ and IL-8 were significantly elevated in the PC group compared to the NC group $(P<0.05)$, but there was no significant difference between the NC and EXP groups, and the same change between $\mathrm{PC}$ and EXP groups $(P>0.05)$ (Figures 3B,C).

\section{C. butyricum Modulated muc2 Expression in Intestines of $S$. Enteritidis-Infected Chickens}

The expression of muc2 in chicken intestines was detected via real-time PCR. The results showed that the expression level of muc2 in the jejunum was decreased in the PC group compared to the EXP groups $(P<0.05)$, but there was no significant difference between the NC and EXP groups, and the same change between PC and NC groups $(P>0.05)$. Of note, $C$. butyricum effectively attenuated the $S$. enteritidis-induced changes to muc2 expression in the jejunum. There were no significant differences in muc2 expression in the duodenum, ileum, or cecum among any of the groups $(P>0.05)$ (Figure 2E). Furthermore, we investigated the effects of $C$. butyricum on the muc2 expression in IECs in vitro, and our data showed that after 2 and $6 \mathrm{~h}$ post-infection, the gene expression level of muc2 was not significantly different among the different groups $(P>0.05)$ (Figure 3E).

\section{C. butyricum Increased Intestinal Barrier Function in S. Enteritidis-Infected Chickens}

In this study, we evaluated the effects of C. butyricum on epithelial barrier function in the chicken intestines by detecting the expression level of Zonula occludens-1 (ZO-1) and Occludin via real-time PCR. The results showed that at 6 days post-infection, the expression level of $\mathrm{ZO}-1$ in duodenum and jejunum was significantly decreased in the PC group compared with the EXP group $(P<0.05)$, but there was no significant difference between the NC and EXP groups, and the same change between PC and NC groups $(P>0.05)$. There were no significant differences in ZO-1 expression in either the ileum or cecum among any of the groups $(P>0.05)$ (Figure $2 \mathrm{~F}$ ). Similarly, no significant difference in Occludin levels was found in intestines among the 

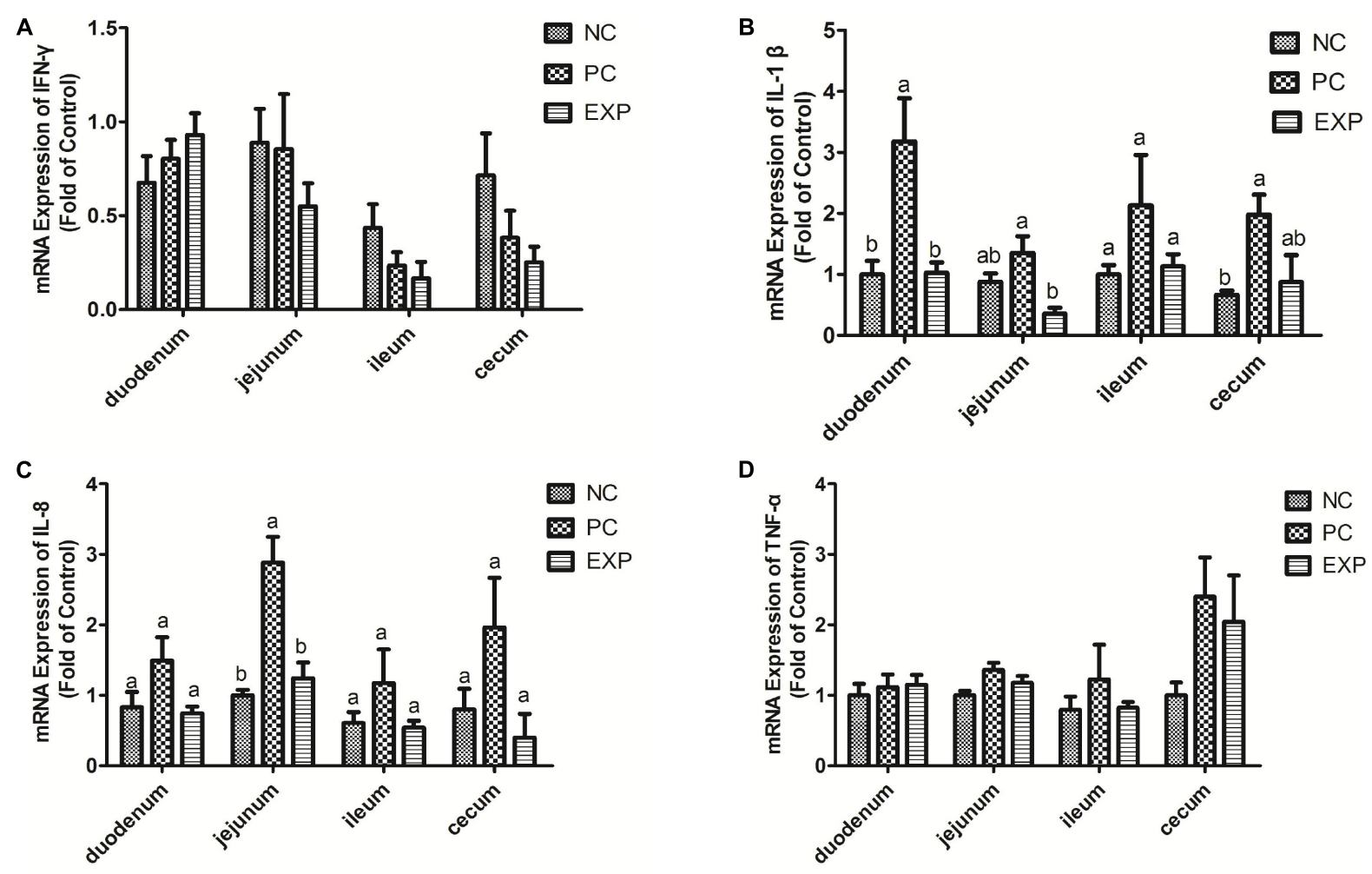

D
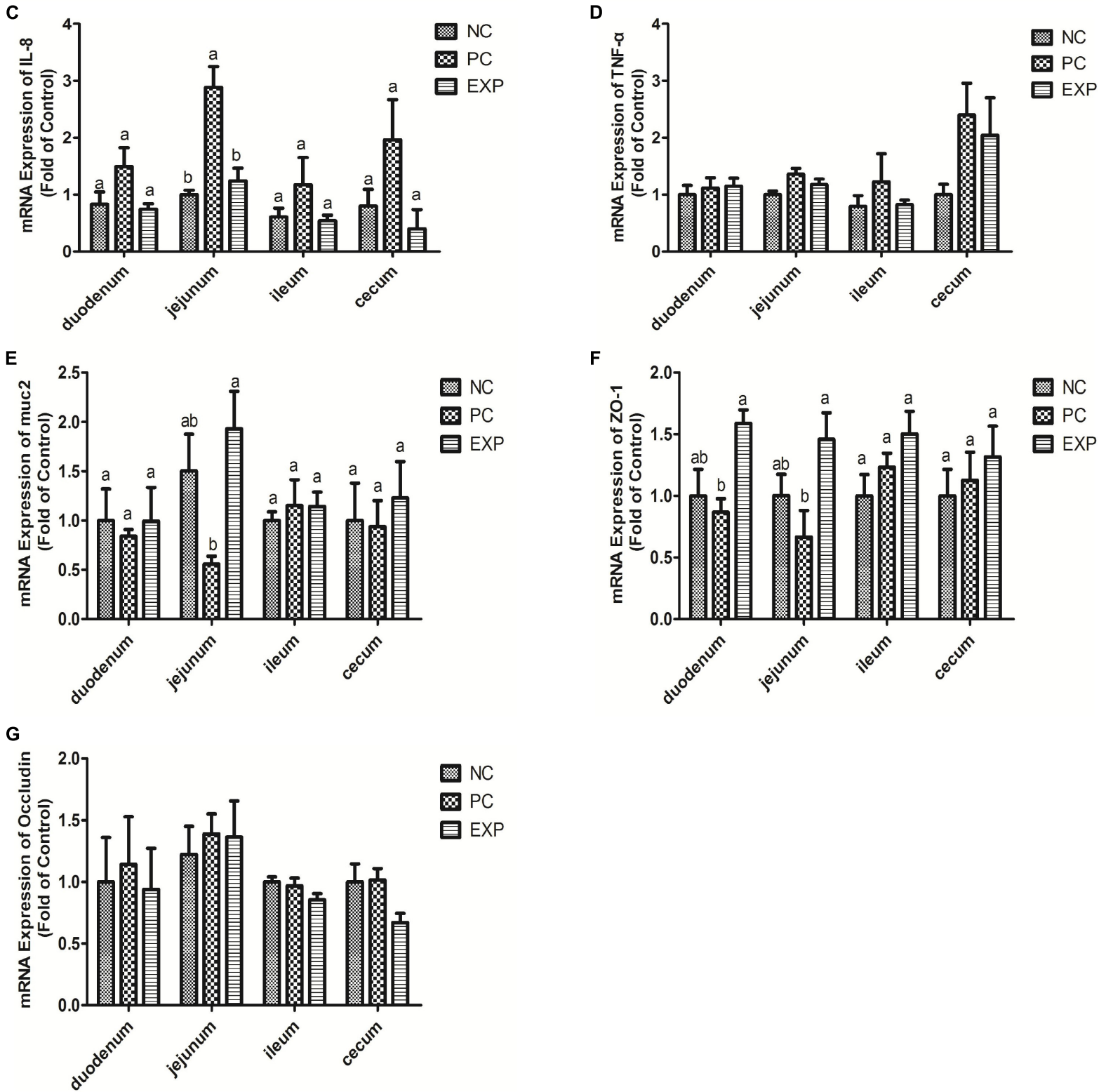

FIGURE 2 | Expression level of cytokines (IFN- $\gamma$, IL-1 $\beta$, IL-8, and TNF- $\alpha$ ), muc2 mucin and the tight junction proteins (ZO-1 and Occludin) (A-G) in intestine tissues (duodenum, jejunum, ileum, and cecum) were estimated by real-time PCR. The bars represent the mean \pm SD ( $n=6 /$ group). Different letters over the bars indicate statistically differences between the groups $(P<0.05)$, same letters over the bars indicate no statistically differences between the groups $(P>0.05)$. NC, the negative control group; PC, the positive control group; EXP, C. butyricum $+S$. enteritidis treatment. 

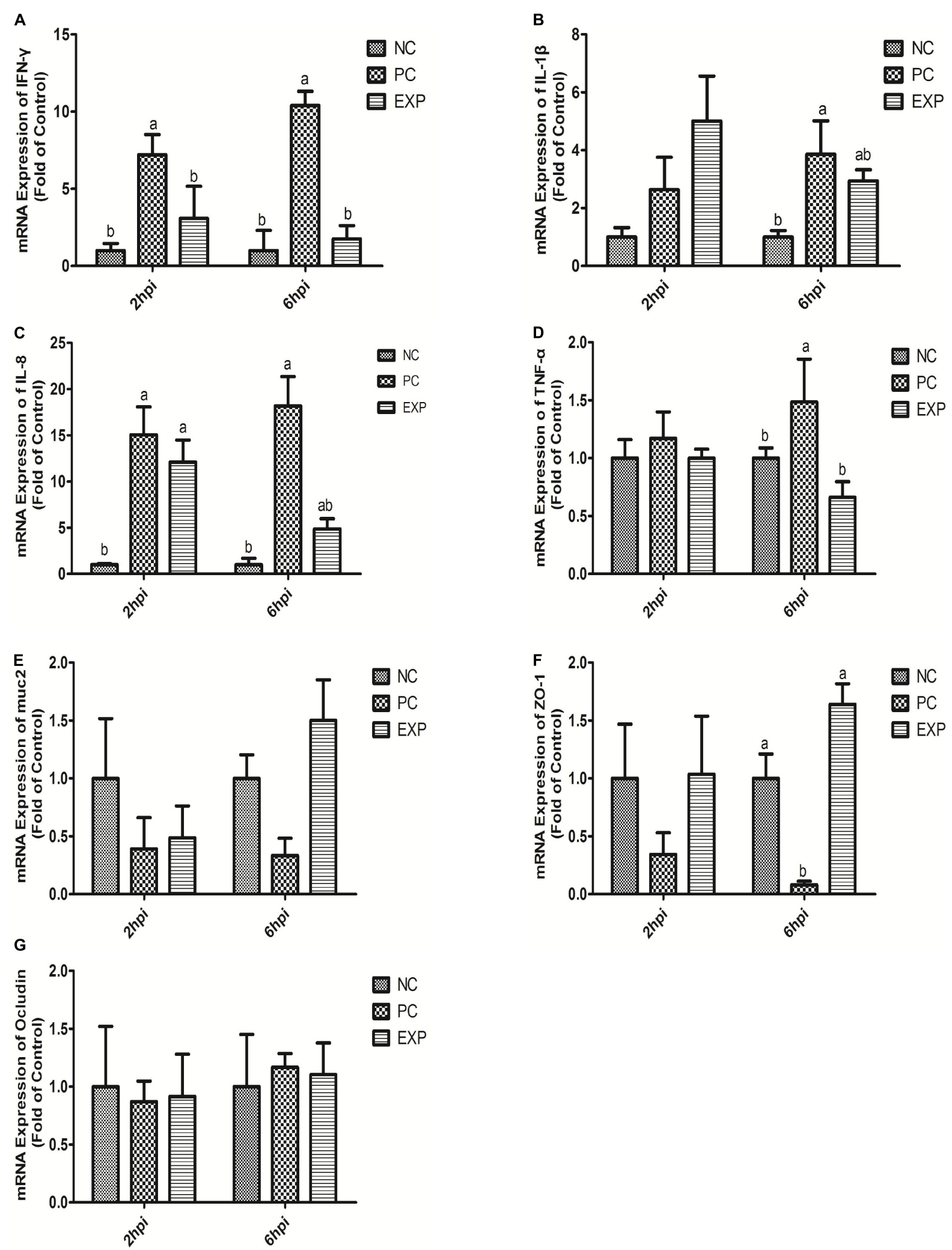

FIGURE 3 | Expression level of cytokines (IFN- $\gamma$, IL-1 1 , IL-8, and TNF- $\alpha$ ), muc2 mucin and the tight junction proteins (ZO-1 and Occludin) (A-G) in intestinal epithelia cells were estimated by real-time PCR. The bars represent the mean $\pm \mathrm{SD}(n=6 /$ group). Different letters over the bars indicate statistically differences between the groups $(P<0.05)$, same letters over the bars indicate no statistically differences between the groups $(P>0.05)$. NC, the negative control group; PC, the positive control group; EXP, C. butyricum + S. enteritidis treatment. 
NC, PC, and EXP groups $(P>0.05)$ (Figure 2G). We also investigated the effects of $C$. butyricum on tight junction (TJ) expression in IECs in vitro. The data show that after $2 \mathrm{~h}$ postinfection, the expression levels of ZO-1 and Occludin were not significantly different among NC, PC and EXP groups $(P>0.05)$ (Figures 3F,G); but after $6 \mathrm{~h}$ post-infection, the expression of ZO1 was significantly decreased in the PC group compared to the EXP group $(P<0.05)$, and there was no significant difference between the NC and EXP groups $(P>0.05)$ (Figure 3F). The expression of Occludin $6 \mathrm{~h}$ post-infection was not significantly different among any of the groups $(P>0.05)$ (Figure 3G).

\section{C. butyricum Suppressed TLR4-, MyD88-, and NF- $\kappa B-D e p e n d e n t$ Inflammation Pathways}

Chickens in the EXP group had decreased gene expressions of TLR4, MyD88, and NF- $\kappa B$ in the jejunum compared to those in the PC group $(P<0.05)$, but there was no significant difference between the NC and EXP groups and the same change between PC and NC groups regarding the gene expressions of MyD88 $(P>0.05)$, which indicates a direct effect of $C$. butyricum. There were no significant differences in TLR4 and MyD88 expression in the duodenum, ileum, or cecum among any of the groups $(P>0.05)$. The expression level of NF-kB in duodenum was significantly elevated in the PC group compared with the EXP and $\mathrm{NC}$ groups $(P<0.05)$, but there was no significant difference between the NC and EXP groups $(P>0.05)$ (Figure 4). We further investigated the effects of C. butyricum on the TLR4, MyD88, and NF- $\mathrm{KB}$ expression levels in IECs in vitro and our results show that, after $2 \mathrm{~h}$ post-infection, the gene expression levels of TLR4, MyD88, and NF- $\mathrm{B}$ were not significantly different among any of the groups $(P>0.05)$ (Figure 5); but after $6 \mathrm{~h}$ post-infection, C. butyricum decreased the gene expression levels of TLR4, MyD88, and NF- $\kappa$ B in the EXP group compared with the PC group $(P<0.05)$, but there was no significant difference between the NC and EXP groups and the same change between PC and NC groups $(P>0.05)$ (Figure 5).

\section{The Effects of C. butyricum on the Bacterial Community Within Chicken Cecum}

We evaluated the effects of $C$. butyricum on the microbiota in chicken cecum using Illumina sequencing of the $16 \mathrm{~S}$ rRNA V4 region. Firmicutes, Tenericutes, and proteobacteria were

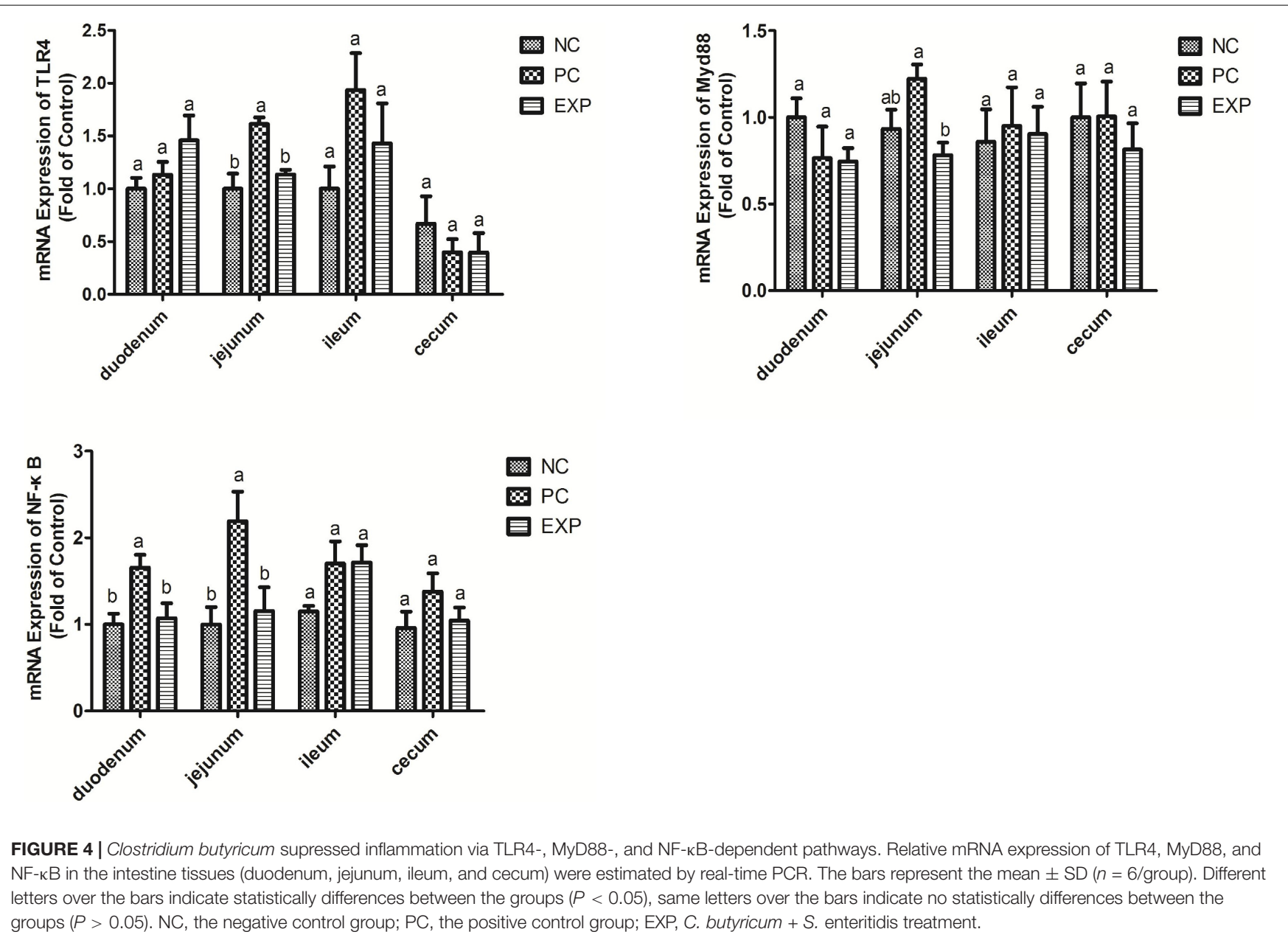




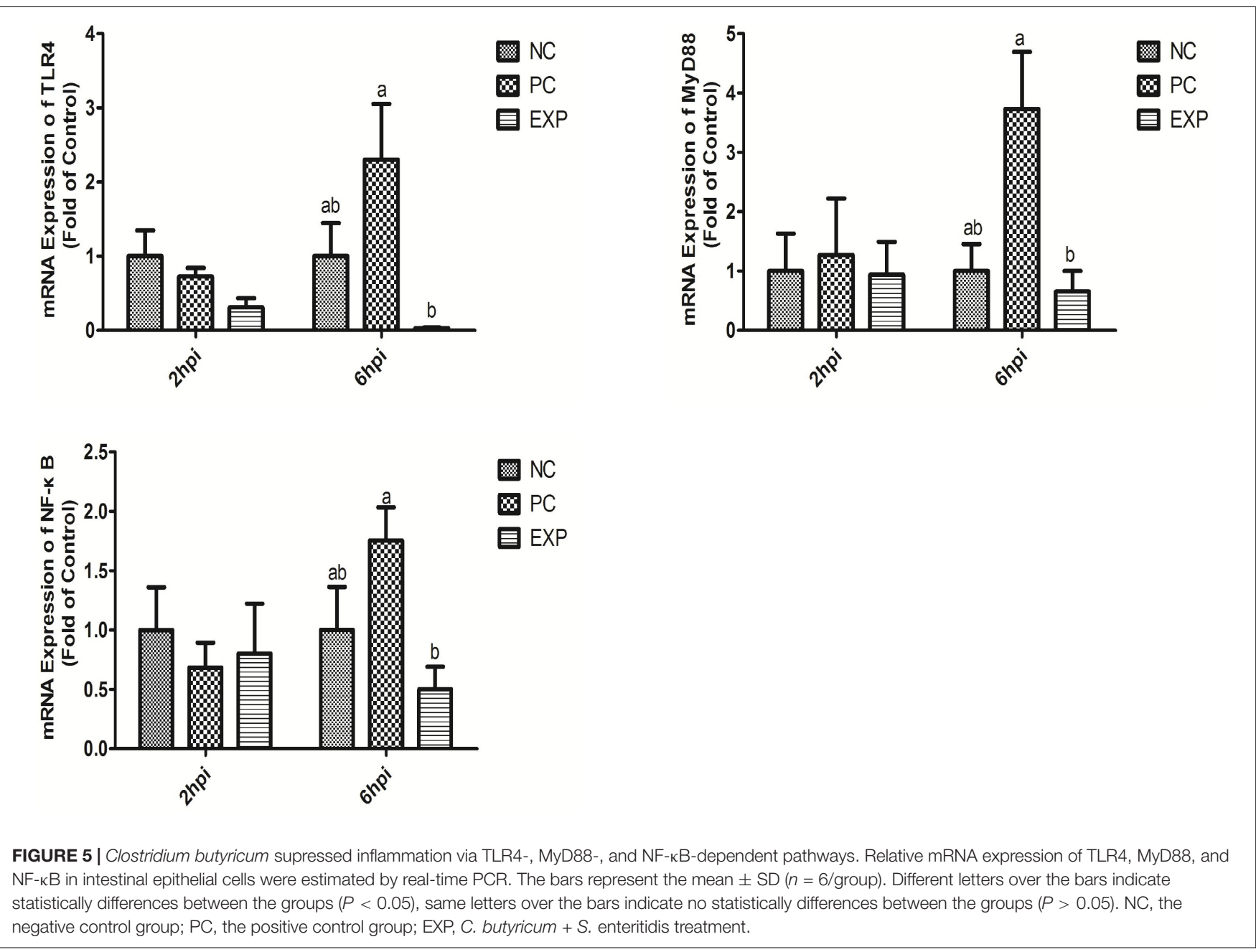

the three most abundant bacterial phyla in all samples, and C. butyricum increased the proportion of Tenericutes in the EXP chickens compared to the NC and PC groups (Figure 6A). The genera Ruminococcus, Oscillospira, Coprococcus, and Dorea were the most prevalent in all of the groups, and the proportion of Coprococcus and Dorea in NC and EXP groups was increased compared to the PC group (Figure 6B). The diversity of the intestinal bacterial community was determined by Shannon, Chaol, and AEC indices. The results show that C. butyricum increased the diversity of the bacterial community in the EXP group compared to the $\mathrm{NC}$ and $\mathrm{PC}$ groups (Figures 6C-E). Collectively, these data suggest that $C$. butyricum affects bacterial composition in the cecum of chickens.

\section{DISCUSSION}

Gram-negative S. enterica was identified as the most common cause of food poising in China (Ran et al., 2011) and is known to disrupt the intestinal epithelial layer during its infection (Coburn et al., 2007). In this study, C. butyricum protected the integrity of the villi in the cecum, limited the invasion of Salmonella; attenuated Salmonella-induced microbiota disruption in the intestine of chickens; improved intestinal epithelial barrier function through the modulation of Muc-2 and ZO-1 expression. Our results suggest that $C$. butyricum is a potential therapy for Salmonella infection or other intestinal diseases.

It has been reported that Salmonella could easily colonize the gut and induce a strong intestinal inflammatory response due to the defective microbial barriers and innate immune systems in the newly-hatched chicks (Brown et al., 2006). In the present study, C. butyricum significantly decreased the expression level of the pro-inflammatory cytokine (IL- $1 \beta$ and IL-8) production in intestines and the expression level of the pro-inflammatory cytokine (IFN- $\gamma$, IL-1 $\beta$, IL-8, and TNF- $\alpha$ ) in intestinal epithelial cells of chickens after Salmonella infection. The protective action of C. butyricum was similar to that of other probiotics (Castillo et al., 2013) and it maybe depended on its antibacterial acticity. Furthermore, we found that C. butyricum suppressed intestinal inflammation by downregulating the TLR4-, MyD88, and NF-кB-dependent pathways in chickens with Salmonella infection, consistent with previous studies that $C$. butyricum can decrease pro-inflammatory cytokine levels by inhibiting the NF-KB signaling pathway in broiler chickens with Salmonella 



FIGURE 6 | The diversity and composition of the bacterial communities were determined by $a$ diversity according to Personalbio's recommendations. (A) Relative abundance of the most abundant bacterial phyla. (B) Relative abundance of the most abundant bacterial genus. The diversity of the bacterial communities were determined by Shannon index (C), Chao1 index (D), ACE index (E). (A-C) Represented sample of NC, PC, and EXP group. NC, the negative control group; PC, the positive control group; EXP, C. butyricum + S. enteritidis treatment.

infection (Zhao et al., 2017). The result suggests the linkage of TLR4/NF-KB pathway may involved in the suppression of C. butyricum on Salmonella infection.

Muc2 is the major gel-forming mucin of the intestine and is the main structural component of the mucus gel. It is generally assumed that muc2 is essential for epithelial protection (Gill et al., 2011). In this study, muc2 production was decreased in the jejunum of chickens with Salmonella infection. However, C. butyricum attenuated the Salmonella-induced disruption of muc2 production, which is consistent with another study that showed supplementation of LGG before and after DON/ZEA exposure appeared to increase muc2 (Murphy et al., 2016), but our results are different than those reported in mice (Gaudier et al., 2005), that mucin gene expression was not altered by 
probiotic administration, this may be due to the differences in probiotic strains.

Tight junctions play a very important role in the intestinal mucosal barrier against macromolecular transmission (Ballard et al., 1995). ZO-1 and Occludin are important proteins responsible for the structural and functional organization of tight junctions (Fanning et al., 1998). In this study, we demonstrated that $C$. butyricum enhanced epithelial barrier function by increasing the expression of ZO-1 in intestinal tissue and IECs infected with Salmonella, which is consistent with a previous report showing that mRNA levels of ZO-1 in broiler chickens fed a 300 or $450 \mathrm{~g} /$ ton $\beta$-mannanase diet were significantly higher (Zuo et al., 2014).

Dietary supplementation of C. butyricum strains as a probiotic has become an effective alternative to the use of antibiotics to increase health and growth performance of chickens, as it has been shown that probiotics can positively affect the gut microbiota, which plays an important role in health and nutrient digestion in chickens (Yang et al., 2012). In this study, we found that $C$. butyricum treatment could alter the intestinal microbial composition and increase the diversity of the bacterial community, which could directly or indirectly impact chicken health and reduce or inhibit the presence of opportunistic pathogens and it may be due to its ability to produce metabolites, which can regulate the $\mathrm{pH}$ (acid change) of intestinal, inhibit pathogenic bacteria, and thus adjust the bacterial community structure. Our study aligns with another study that showed a diet supplemented with Enterococcus faecalis could shift microbial diversity in the porcine gut and inhibit pathogens (Li et al., 2016).

\section{CONCLUSION}

Clostridium butyricum effectively attenuated inflammation and epithelial barrier damage, altered the intestinal microbial composition by increasing the diversity of the bacterial

\section{REFERENCES}

Ballard, S. T., Hunter, J. H., and Taylor, A. E. (1995). Regulation of tight-junction permeability during nutrient absorption across the intestinal epithelium. Annu. Rev. Nutr. 15, 35-55. doi: 10.1146/annurev.nu.15.070195.000343

Brown, S. P., Cornell, S. J., Sheppard, M., Grant, A. J., Maskell, D. J., and Mastroeni, P. (2006). Intracellular demography and the dynamics of Salmonella enterica infections. PLoS Biol. 4:349. doi: 10.1371/journal.pbio.0040349

Cao, G. T., Xiao, Y. P., Yang, C. M., Chen, A. G., Liu, T. T., Zhou, L., et al. (2012). Effects of Clostridium butyricum on growth performance, nitrogen metabolism, intestinal morphology cecal microflora in broiler chickens. J. Anim. Vet. Adv. 11, 2665-2671. doi: 10.3923/javaa.2012.2665.2671

Castillo, N. A., de Moreno de LeBlanc, A., Galdeano, M. C., and Perdigón, G. (2013). Comparative study of the protective capacity against Salmonella infection between probiotic and nonprobiotic lactobacilli. J. Appl. Microbiol. 114, 861-876. doi: 10.1111/jam.12074

Chen, Z., and Jiang, X. (2014). Microbiological safety of chicken litter or chicken litter-based organic fertilizers: a review. Agriculture 4, 1-29. doi: 10.3390/ agriculture 4010001

Coburn, B., Grassl, G. A., and Finlay, B. B. (2007). Salmonella, the host and disease: a brief review. Immunol. Cell Biol. 85, 112-118. doi: 10.1038/sj.icb.7100007 community, and promoted immune function in the intestines of Salmonella-infected chicken. C. butyricum might be an effective and safe therapy for Salmonella infection.

\section{Future Work}

In future work, we will supplement the detection of Salmonella and Clostridium butyricum counts during the course of the experiments to further verify that the organism of the bacteria colonized the gut.

\section{DATA AVAILABILITY STATEMENT}

The datasets generated for this study are available on request to the corresponding author.

\section{ETHICS STATEMENT}

The animal study was reviewed and approved by The Animal Care and Use Committee of Shandong Agricultural University.

\section{AUTHOR CONTRIBUTIONS}

HL, SS, and XZ conceived and designed the study. XZ, JY, ZJ, JW, and LW performed the experiments and analyzed the data. HL, $\mathrm{SS}$, and $\mathrm{XZ}$ wrote and revised the manuscript.

\section{FUNDING}

This work was supported by the National Key R\&D Project (2016YFD0501608 and 2016 YFD0500510), Taishan Scholar Program (201511023), Funds of Shandong "Double Tops" Program, and Shandong Agricultural Major Applied Technology Innovation Project (SD2019XM009).

Fanning, A. S., Jameson, B. J., Jesaitis, L. A., and Anderson, J. M. (1998). The tight junction protein $\mathrm{ZO}-1$ establishes a link between the transmembrane protein occludin and the actin cytoskeleton. J. Biol. Chem. 273, 29745-29753. doi: 10.1074/jbc.273.45.29745

Feasey, N. A., Dougan, G., Kingsley, R. A., Heyderman, R. S., and Gordon, M. A. (2012). Invasive nontyphoidal Salmonella disease: an emerging and neglected tropical disease in Africa. Lancet 379, 2489-2499. doi: 10.1016/S0140-6736(11) 61752-2

Forstner, J., Oliver, M., and Sylvester, F. (1995). Production, Structure and Biologic Relevance of Gastrointestinal Mucins. New York, NY: Raven Press.

Gaudier, E., Michel, C., Segain, J. P., Cherbut, C., and Hoebler, C. (2005). The VSL\# 3 probiotic mixture modifies microflora but does not heal chronic dextransodium sulfate-induced colitis or reinforce the mucus barrier in mice. J. Nutr. $135,2753-2761$.

Gill, N., Wlodarska, M., and Finlay, B. B. (2011). Roadblocks in the gut: Barriers to enteric infection. Cell Microbiol. 13, 660-669. doi: 10.1111/j.1462-5822.2011. 01578.x

Huang, Q. Q., Wei, Y. N., Lv, Y. J., Wang, Y. X., and Hu, T. M. (2015). Effect of dietary inulin supplements on growth performance and intestinal immunological parameters of broiler chickens. Livest Sci. 180, 172-176. doi: 10.1016/j.livsci.2015.07.015 
Juan, D. L., Adhikari, B., Park, S. H., Teague, K. D., Graham, L. E., Mahaffey, B. D., et al. (2018). Evaluation of the epithelial barrier function and ileal microbiome in an established necrotic enteritis challenge model in broiler chickens. Front. Vet. Sci. 5:199. doi: 10.3389/fvets.2018.00199

Kemgang, S. T., Kapila, S., Shanmugam, V. P., and Kapila, R. (2014). Cross-talk between probiotic lactobacilli and host immune system. J. Appl. Microbiol. 117, 303-319. doi: 10.1111/jam.12521

Kusumawati, D. I., Harmayani, E., and Asmara, W. (2006). Effect of probiotic lactobacillus sp. Dad 13 on humoral immune response of balb/C mice infected with Salmonella typhimurium, Indonesian. J. Biotechnol. 11, 870-877.

Li, P. H., Niu, Q., Wei, Q. T., Zhang, Y. Q., Ma, X., Kim, S. W., et al. (2016). Microbial shifts in the porcine distal gut in response to diets supplemented with Enterococcus Faecalis as alternatives to antibiotics. Sci. Rep. 7:41395. doi: $10.1038 /$ srep41395

Lim, S. M., Jang, H. M., Jeong, J. J., Han, M. J., and Kim, D. H. (2017). Lactobacillus johnsonii CJLJ103 attenuates colitis and memory impairment in mice by inhibiting gut microbiota lipopolysaccharide production and NF- $\kappa \mathrm{B}$ activation. J. Funct. Foods 34, 359-368. doi: 10.1016/j.jff.2017.05.016

Madsen, K. L. (2012). Enhancement of epithelial barrier function by probiotics. J. Epithelial Biol. Pharmacol. 5, 55-59. doi: 10.2174/1875044301205010055

Majowicz, S. E., Musto, J., Scallan, E., Angulo, F. J., Kirk, M., O’Brien, S. J., et al. (2010). The global burden of nontyphoidal Salmonella gastroenteritis. Clin. Infect Dis. 50, 882-889. doi: 10.1086/650733

Mathur, R., Oh, H., Zhang, D., Park, S. G., Seo, J., Koblansky, A., et al. (2012). A mouse model of Salmonella typhi infection. Cell 151, 590-602. doi: 10.1016/j. cell.2012.08.042

Murphy, L. Y. W., Paul, C. T., Kevin, J. A., and Hani, E. N. (2016). Lactobacillus rhamnosus GG modulates intestinal mucosal barrier and inflammation in mice following combined dietary exposure to dexy nivalenol and zearalenone. J. Funct. Foods 22, 34-43. doi: 10.1016/j.jff.2016.01.014

Parry, C. M., and Threlfall, E. J. (2008). Antimicrobial resistance in typhoidal and nontyphoidal Salmonellae. Curr. Opin. Infect. Dis. 21, 531-538. doi: 10.1097/ QCO.0b013e32830f453a

Pierzchalska, M., Grabacka, M., Michalik, M., Zyla, K., and Pierzchaski, P. (2012). Prostaglandin E2 supports growth of chicken embryo intestinal organoids in Matrigel matrix. Biotechniques 52, 307-315. doi: 10.2144/0000113851

Ran, L., Wu, S., Gao, Y., Zhang, X., Feng, Z., Wang, Z., et al. (2011). LaboratoryBased Surveillance of Nontyphoidal Salmonella infections in China. Foodborne Pathog. Dis. 8, 921-927. doi: 10.1089/fpd.2010.0827

Sainte-Marie, G. A. (1962). Paraffin embedding technique for studies employing immunofluorescence. J. Histochem. Cytochem. 10, 150-156.

Schloss, P. D., Westcott, S. L., Ryabin, T., Hall, J. R., Hartmann, M., Hollister, E. B., et al. (2009). Introducing mothur: open-source, platform-independent, community-supported software for describing and comparing microbial communities. App. Environ. Microb. 75, 7537-7541. doi: 10.1128/AEM. 01541-09

Shanahan, F. (2010). Probiotics in perspective. Gastroenterology 139, 1808-1812. doi: 10.1053/j.gastro.2010.10.025

The European Pharmacopoeia 7. 0, (2010). General Text 5.2.2. Chicken Flocks Free From Specified Pathogens for the Production and Quality Control of Vaccines. Strasbourg: The European Pharmacopoeia commission, 527-530.

Wei, C. L., Chao, S. H., Tsai, W. B., Lee, P. S., Tsau, N. H., Chen, J. S., et al. (2013). Analysis of bacterial diversity during the fermentation of inyu, a hightemperature fermented soy sauce, using nested PCR-denaturing gradient gel electrophoresis and the plate count method. Food Microbiol. 33, 252-261. doi: 10.1016/j.fm.2012.10.001

Xiao, M., Mi, Y., Liu, L., Lv, C., Zeng, W., Zhang, C., et al. (2018). Taurine regulates mucosal barrier function to alleviate lipopolysaccharide-induced duodenal inflammation in chicken. Amino Acids. 50, 1637-1646. doi: 10.1007/s00726018-2631-6

Yang, C. M., Cao, G. T., Ferket, P. R., Liu, T. T., Zhou, L., Zhang, L., et al. (2012). Effects of probiotic, Clostridium butyricum, on growth performance, immune function, and cecal microflora in broiler chickens. Poult. Sci. 91, 2121-2129. doi: 10.3382/ps.2011-02131

Zhang, L., Zhang, L., Zhan, X., Zeng, X., Zhou, L., Cao, G., et al. (2016). Effects of dietary supplementation of probiotic, Clostridium butyricum, on growth performance, immune response, intestinal barrier function, and digestive enzyme activity in broiler chickens challenged with Escherichia coli K88. J. Anim. Sci Biotechnol. 7, 107-115. doi: 10.1186/s40104-016-0061-4

Zhao, X. N., Yang, J., Wang, L. L., Lin, H., and Sun, S. H. (2017). Protection mechanism of Clostridium butyricum against Salmonella enteritidis infection in broilers. Front. Microbiol. 8:1523. doi: 10.3389/fmicb.2017. 01523

Zuo, J. J., Guo, A. H., Yan, X. Y., Xu, M., Xia, W. G., and Feng, D. Y. (2014). Supplementation of $\beta$-Mannanase in diet with energy adjustmenton affect performance, intestinal morphology and tight junction proteins mRNA expression in broiler chickens. J. Anim. Vet. Adv. 13, 144-151.

Conflict of Interest: The authors declare that the research was conducted in the absence of any commercial or financial relationships that could be construed as a potential conflict of interest.

Copyright (c) 2020 Zhao, Yang, Ju, Wu, Wang, Lin and Sun. This is an open-access article distributed under the terms of the Creative Commons Attribution License (CC BY). The use, distribution or reproduction in other forums is permitted, provided the original author(s) and the copyright owner(s) are credited and that the original publication in this journal is cited, in accordance with accepted academic practice. No use, distribution or reproduction is permitted which does not comply with these terms. 